\title{
Traffic Congestion Estimation for Short-Highway in Pre- Timed Systems
}

\author{
Arthit Buranasing and Chaiyaporn Khemapatapan
}

\begin{abstract}
Only a single CCTV camera is installed on highway, this is cause of difficulties to automated estimation of traffic congestion. Many factors are hidden from the data input. For example, arrival-flow/departure-flow rates, traffic light cycles and etc. This paper studies the effect of primary hidden factors to traffic congestion on a single CCTV camera for highway in pre-timed systems. Some characteristics are revealed with significance to the estimation of traffic congestion. A model for traffic congestion is proposed. Comprehensive experiments are presented. The technique can estimate traffic congestion for real-time systems with low error rate.
\end{abstract}

Index Terms - Traffic congestion estimation, queue length estimation, intelligent transportation system (ITS), highway engineering, traffic signal management system.

\section{INTRODUCTION}

Traffic congestion is one of the most important measures for evaluating the performance of traffic network systems and also important to traveler for decisions on travel choices and avoid unnecessary delay. However, the majority of works on traffic congestion estimation are on freeway, where no traffic light is involved in the system and most of those works has much cost. Highway, on the other hand, is more complicated as traffic light cycles play an important role and many factors are hidden in the system and affect to traffic congestion [1].

So far, works on traffic congestion for highway have been conducted with different techniques and different constraints of input data. For example, Bernhard Krause, Constantin von Altrock and Martin Pozybill [2] used two detectors which were installed at the beginning of the road and the end of the road for detection traffic volume and vehicles average velocity for prediction traffic congestion on multilane road by using fuzzy logic. The model performed well, but the model had much cost and complicated for real-time systems. Yasushi Ando, Osamu Masutani and Shinichi Honiden [3] developed traffic congestion prediction model with Pheromone technique on simulator by installing GPS devices in each vehicle for collecting traffic data (or probevehicles systems) such as average velocity on the road for a input data in theirs model. In this work, the technique performed well on static traffic, but not good on fluctuated traffic. Recently, Arthit Buranasing and Akara Prayote [1]

Manuscript received January 13, 2013; revised March 27, 2013.

The authors are with Faculty of Engineering, Dhurakij Pundit University, Bangkok, Thailand (e-mail: \{arthit.bur, chaiyaporn\}@dpu.ac.th). developed traffic congestion estimation model for highway by using only flow rate and average velocity in each minute from a single CCTV camera which is installed in the middle of the road without using traffic signal data or departureflow rate on the pre-timed system. However, the model could only predict traffic congestion on un-congested condition, but could not predict traffic congestion on congested condition.

Hence, so far to the best of our knowledge, traffic congestion estimation on highway requires at least two type of raw data, one is the data from a CCTV camera which some work requires at least two cameras and the others is traffic signal data or departure-flow rate. Although, Buranasing and Prayote attempted to develop traffic congestion model for highway by using only a single camera for saving cost, but the model could not predict traffic congestion on congested condition. Therefore, in this paper, the proposed model are able to estimate traffic congestion on short-highway by using only average velocity from a single camera which is installed at the beginning of the road by without using traffic signal data or departure-flow rate on the pre-timed system and the model are also able to predict traffic congestion in any traffic condition (both congested and un-congested condition). This paper is organized into the following sections: Section II simulation software and experiment setting. Equivalent queue length estimation model in Section III. Characteristic of CCTV's velocity in Section IV. Velocity and congestion model in section V. Traffic congestion estimation model and performance in section VI and a conclusion and remark is drawn in section VII.

\section{Simulation Software AND ExPERIMENT SETting}

Quadstone Paramics Simulation Software [4] was used for the experiment testing and used some major simulation setting which was derived from Buranasing and Prayote. (Quadstone Paramics Simulation Software was also used in theirs proposes.)

In this proposes, the experiments were defined as follows; used 2 type of roads which each road had length $0.5 \mathrm{~km}$. and $1.0 \mathrm{~km}$. with 1 lane, set vehicle only 4 wheels cars which each car had 4 meters length and 1 meter for suitable gap, maximum vehicle speed was $120 \mathrm{~km} / \mathrm{h}$, traffic was controlled by pre-timed system which each experiment had a cycle length 60, 120 and 240 seconds and used green time per red time $25 \%, 50 \%$ and $75 \%$ in each cycle length experiment. (For instance, green time $25 \%$ of 120 seconds cycle length, means green time 30 seconds and red time 90 seconds. Yellow time was included in green time, about 5 seconds in each green time). In simulation, collection data 
was derived from a single CCTV camera which was installed at the beginning of the road and detected average velocity in each minute and also detected average congestion by using function of simulator. Then, launched simulator by started from empty road and released vehicles to the road and controlled arrival-flow rate more than departure-flow rate until road was full (road had a maximum congestion) within first 180 minutes and next 180 minutes later controlled arrival-flow less than departure-flow until the road was empty again, each experiment had the same this scenario. In simulation excluded exception-event such as accident, using siren of vehicle, human cross a road and etc, vehicles flows were run normally. The data from simulation experiment will be used for testing all of the rest in this paper.

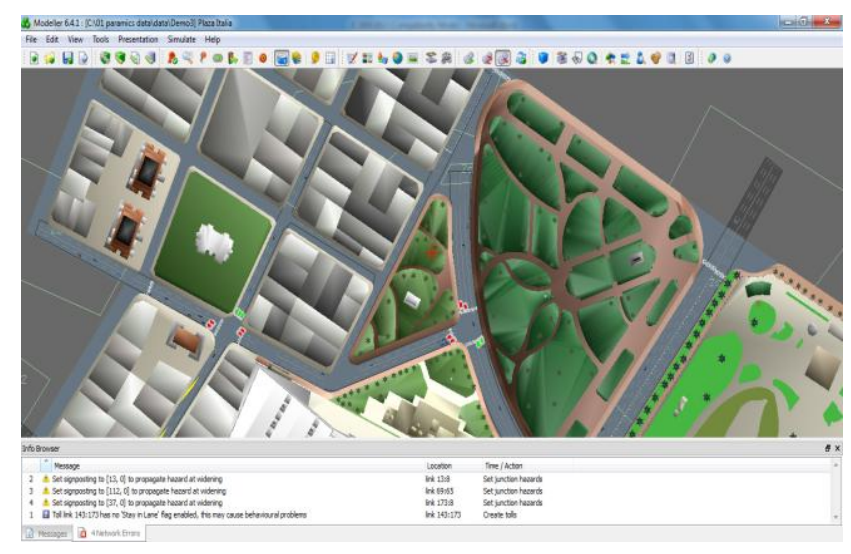

Fig. 1. Traffic Simulations on Quadstone Paramics.

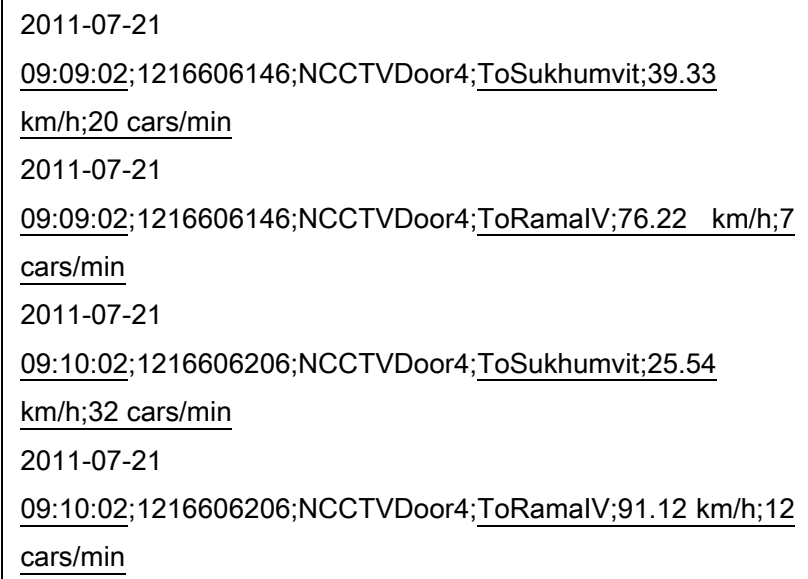

Fig. 2. Sample's data from CCTV camera.

\section{EQuiVALENT QUEUE LENGTH ESTIMATION MODEL}

The techniques and some equation of equivalent queue length estimation model [1] were involved in traffic congestion estimation model in this paper and the model can estimate as follows.

The equivalent queue length can be estimated in sequence as follows.

$$
V_{\mathrm{ma}}=\frac{\sum_{i=1}^{T} \mathrm{~V}_{\mathrm{CCTV}_{\mathrm{i}}}}{T}
$$

where $V_{\text {CCTV }_{i}}$ is velocity from CCTV camera in each minute, $V_{\text {ma }}$ is an average velocity which is computed in time window size of $T=15$ minutes, $\mathrm{T}$ is time window size.

$$
Q=C \sqrt{1-\frac{V_{\mathrm{ma}}}{V_{\max }}}
$$

where $Q$ is an equivalent queue length, $C$ is CCTV position, $V_{\max }$ is a maximum speed that vehicle can reach on an empty road.

A queue's length can be translated into the amount of vehicles accumulated or congestion by

$$
K=\frac{Q}{\mathrm{AVL}}
$$

where AVL is the length of space a vehicle occupies when stopping, and can be calculated as follow.

$$
\mathrm{AVL}=\frac{\sum_{i=1}^{N} \mathrm{LVT}_{i}+\sum_{i=1}^{N} \mathrm{MG}_{i}}{N}
$$

where LVT is a length of vehicle in each type, MG is minimum gap between 2 vehicles, $N$ is number of vehicle's type in the system.

However, $K$ is set to 0 , when no vehicle passes and $V$ is zero [1]. In addition, maximum congestion on a road can be calculated as follow.

$$
K_{\max }=\frac{\mathrm{RL}}{\mathrm{AVL}}
$$

where $K_{\max }$ is maximum congestion, $R$ is road length, $L$ is number of lane.

\section{CHARACTERISTIC OF CCTV'S VELOCITY}

In this model, CCTV camera which was installed at the beginning of the road is cause of low-velocity from the detector whereas vehicle has just started accelerate from previous intersection to next link of the road and cannot reach the maximum speed at the short distance (distance between intersection and CCTV camera), despite of empty road or un-congested on traffic condition.

Nevertheless, from the experiment by using observer method [5], the velocity from the CCTV camera which was reduced will approximately be 2.0 to 3.0 time of maximum speed. Furthermore, low-velocity must multiply less than high-velocity, whereas low-velocity is possibility of congested condition and should not increase too much. As a result, velocity proportion can be calculated as follow.

$$
V_{\beta}=\alpha \sqrt{\frac{V_{\mathrm{ma}}}{V_{\max }}}
$$

where $V_{\beta}$ is a velocity proportion of vehicles, $\alpha$ is a constant of multiplier (2.5 was suitable value for the model and was used for the experiment testing), $\mathrm{V}_{\mathrm{ma}}$ is an average velocity 
which is computed in time window size of $T=15$ minutes, $V_{\max }$ is a maximum speed that a vehicle can reach on an empty road.

As a consequence, the approximately average velocity that vehicle is able to run throughout the road can be form as follow.

$$
V_{f}=V_{\beta} \times V_{\mathrm{ma}} \quad \text { When } V_{f} \leq V_{\max }
$$

where $V_{f}$ is an approximately average velocity that vehicle is able to run throughout the road, $V_{\beta}$ is a velocity proportion of vehicles, $V_{\max }$ is a maximum speed that vehicle can reach on an empty road.

However, some minor velocity's value might more than maximum speed that a vehicle can reach on an empty road, but we can eliminate as follow.

$$
V_{f}=V_{\max } \quad \text { When } \quad V_{f}>V_{\max }
$$

where $V_{f}$ is an approximately average velocity that vehicle is able to run throughout the road, $V_{\max }$ is a maximum speed that vehicle can reach on an empty road.

\section{Velocity AND CONGESTION Model}

Velocity from the CCTV camera is significant input data for the model, because velocity is able to predict congestion on the road. Normally, whenever the road is empty or not much vehicles, vehicles will normally run fast. On the contrary, if road is quite congested, vehicles will run slowly.

Thus, from the experiments by using Pearson's correlation coefficient for evaluated correlation and relationship between 2 parameters, CCTV's velocity and real congestion on $0.5 \mathrm{~km}$ and $1.0 \mathrm{~km}$ road length, it gives a negative correlation with average value -0.97 and -0.97 respectively in all experiment and each experiment are shown in Table I and Table II.

TABLE I: SUMMARY's PEARSON CORRELATION OF CCTV'S VELOCITY

\begin{tabular}{|c|c|c|c|c|c|c|}
\hline \multirow{2}{*}{ GL } & \multicolumn{2}{|c|}{$25 \%$} & \multicolumn{2}{|c|}{$50 \%$} & \multicolumn{2}{c|}{$75 \%$} \\
\cline { 2 - 7 } & $1^{\text {st }}$ & $2^{\text {nd }}$ & $1^{\text {st }}$ & $2^{\text {nd }}$ & $1^{\text {st }}$ & $2^{\text {nd }}$ \\
\hline 60 (Second) & -0.98 & -0.99 & -0.98 & -0.99 & -0.98 & -0.99 \\
\hline 120 (Second) & -0.97 & -0.99 & -0.98 & -0.99 & -0.97 & -0.92 \\
\hline 240 (Second) & -0.98 & -0.99 & -0.97 & -0.98 & -0.98 & -0.99 \\
\hline Average & \multicolumn{7}{|c}{ All $1^{\text {st }}$ average is -0.97} & \multicolumn{1}{c|}{ All $2^{\text {nd }}$ average is -0.98} \\
\hline
\end{tabular}

TABLE II: SUMMARY'S PEARSON CORRELATION OF CCTV's VELOCITY

\begin{tabular}{|c|c|c|c|c|c|c|}
\hline \multirow{2}{*}{ CL } & \multicolumn{2}{|c|}{$25 \%$} & \multicolumn{2}{|c|}{$50 \%$} & \multicolumn{2}{|c|}{$75 \%$} \\
\hline & $1^{\text {st }}$ & $2^{\text {nd }}$ & $1^{\text {st }}$ & $2^{\text {nd }}$ & $1^{\text {st }}$ & $2^{\text {nd }}$ \\
\hline 60 (Second) & -0.99 & -0.99 & -0.97 & -0.99 & -0.96 & -0.99 \\
\hline 120 (Second) & -0.95 & -0.99 & -0.99 & -0.98 & -0.94 & -0.99 \\
\hline 240 (Second) & -0.94 & -0.99 & -0.98 & -0.99 & -0.99 & -0.96 \\
\hline Average & \multicolumn{3}{|c|}{ All $1^{\text {st }}$ average is -0.96} & \multicolumn{3}{|c|}{ All $2^{\text {nd }}$ average is -0.98} \\
\hline
\end{tabular}
AND CONGESTION ON 1.0 KM ROAD LENGTH.

In the Table, CL is cycle length and GTP is green time proportion. Note that $1 \mathrm{st}$ part is the experiment that starts from empty road to road is full in 180 minutes and $2^{\text {nd }}$ part is the experiment that starts from road is full to empty road in 180 minutes.

As a result, CCTV-velocity and congestion model can be formed as follow.

$$
K_{i}=K_{\max }\left[1-\left(V_{f} / V_{\max }\right)\right]
$$

where $K_{i}$ is congestion at time i, $K_{\max }$ is maximum congestion, $V_{f}$ is an approximately average velocity that vehicle is able to run throughout the road, $V_{\max }$ is a maximum speed that vehicle can reach on an empty road.

Nonetheless, for smoothing result, the model used moving average techniques for the last procedure which used time windows $T=15$ minutes (Therefore, the model need about 30 minutes to initial first prediction, first 15 minutes is for CCTV's velocity moving average and next 15 minute to predict traffic congestion by using moving average), the moving average of congestion at time $\mathrm{i}$ can be calculated as follow.

$$
K_{t}=\sum_{i=1}^{T} K_{i} / T
$$

where $K_{\mathrm{t}}$ is an average congestion which is computed in time window size of $T=15$ minutes or average congestion at time $t, T$ is time window size and $K_{i}$ is a congestion at time $i$.

\section{TRAfFic Congestion Estimation Model AND PERFORMANCE}

Some equation of equivalent queue length estimation model (1), (4) and (5) and all equation in this paper, (6) to (10) be rewrite into the sequence of traffic congestion estimation model which is shown below in figure 3

The accuracy of traffic congestion estimation model is also evaluated in 2 parts, one is launch simulator by starts from empty road and release vehicles to the road and control arrival-flow rate more than departure-flow rate until road is full (road has a maximum congestion) within first 180 minutes, this part give an average error $2.49 \mathrm{veh} / \mathrm{km}$ and $4.83 \mathrm{veh} / \mathrm{km}$ on $0.5 \mathrm{~km}$ and $1.0 \mathrm{~km}$ road length respectively and the other 180 minutes part is vice versa which give an average error $2.76 \mathrm{veh} / \mathrm{km}$ and $5.35 \mathrm{veh} / \mathrm{km}$. Both experiments parts on each road length give an average error $2.62 \mathrm{veh} / \mathrm{km}$ on $0.5 \mathrm{~km}$ road length and an average error $5.09 \mathrm{veh} / \mathrm{km}$ on $1.0 \mathrm{~km}$ road length which are shown in table III and table IV, graph in each experiment are shown below the table. (Average error of both $0.5 \mathrm{~km}$ and $1.0 \mathrm{~km}$ road length is $3.85 \mathrm{veh} / \mathrm{km})$.

Time's scale on experiment graph is an only time when queue's length is almost equal maximum road's congestion and vice versa in $2^{\text {nd }}$ experiment, most of them take time about 140 minutes to reach a maximum congestion and empty the road.

Note that an average error $(\mathrm{E})$ in each experiment is value of different between real congestion $(\mathrm{veh} / \mathrm{km})$ and traffic congestion estimation model in each minutes $(\mathrm{T})$, and in this simulator experiment is used about 180 minutes in each experiment testing $(\mathrm{N})$, if value of an error is close to 0 , it will have a high accuracy, whereas the traffic congestion estimation model can predict close to the real congestion. An average error in each experiment testing can be 
calculated as follow.



Fig. 3. Traffic Congestion Estimation Model.

$$
\mathrm{E}=\sum_{\mathrm{T}=1}^{\mathrm{N}} \mid \text { Real Congestion }_{\mathrm{T}}-\text { Congestion Estimation }_{\mathrm{T}} \mid
$$

TABLE III: SUMMARY's ERROR OF TRAFFIC CONGESTION PREDICTION ON 0.5 KM ROAD LENGTH

\begin{tabular}{|c|c|c|c|c|c|c|}
\hline \multirow{2}{*}{ GTP } & \multicolumn{2}{|c|}{$25 \%$} & \multicolumn{2}{c|}{$50 \%$} & \multicolumn{2}{c|}{$75 \%$} \\
\cline { 2 - 7 } & $1^{\text {st }}$ & $2^{\text {nd }}$ & $1^{\text {st }}$ & $2^{\text {nd }}$ & $1^{\text {st }}$ & $2^{\text {nd }}$ \\
\hline 60 (Second) & 2.35 & 1.92 & 2.33 & 2.10 & 2.84 & 3.22 \\
\hline 120 (Second) & 2.46 & 3.41 & 1.63 & 2.55 & 4.24 & 5.86 \\
\hline 240 (Second) & 1.81 & 1.86 & 1.64 & 2.23 & 3.12 & 1.76 \\
\hline Average & \multicolumn{2}{|c|}{ All $1^{\text {st }}$ average is 2.49} & \multicolumn{2}{|c|}{ All $2^{\text {nd }}$ average is 2.76} \\
\hline
\end{tabular}

TABLE IV: SUMMARY's ERROR OF TRAFFIC CONGESTION PREDICTION ON

\begin{tabular}{|c|c|c|c|c|c|c|}
\hline \multicolumn{7}{|c|}{ 1.0 KM ROAD LENGTH } \\
\hline \multirow[t]{2}{*}{ GTP } & \multicolumn{2}{|c|}{$25 \%$} & \multicolumn{2}{|c|}{$50 \%$} & \multicolumn{2}{|c|}{$75 \%$} \\
\hline & $1^{\mathrm{st}}$ & $2^{\text {nd }}$ & $1^{\mathrm{st}}$ & $2^{\text {nd }}$ & $1^{\mathrm{st}}$ & $2^{\text {nd }}$ \\
\hline 60 (Second) & 6.01 & 5.54 & 5.09 & 5.30 & 5.93 & 5.73 \\
\hline 120 (Second) & 2.48 & 6.69 & 5.61 & 5.93 & 2.84 & 2.47 \\
\hline 240 (Second) & 3.59 & 5.29 & 8.54 & 5.49 & 3.41 & 5.79 \\
\hline Average & All 1 & erag & 4.83 & All 2 & averas & s 5.35 \\
\hline
\end{tabular}

\section{CONCLUSION AND REMARK}

This paper studies the effect of primary factors to traffic congestion and was used to develop the model for traffic congestion by using only average velocity from a single camera which is installed at the beginning of the road as input without using traffic signal data on the pre-timed system. In this model experiment exclude exception-event such as accident, using siren of vehicle, human cross a road and etc, vehicles flows are run normally. The result of experiment is satisfy and gives average error or different only $3.85 \mathrm{veh} / \mathrm{km}$ from real congestion. However, the model is not suitable for the long-highway or the road length is not over $1.0 \mathrm{~km}$, whereas velocity which is derived from CCTV camera is not affected by the congestion in the end of the road until vehicles are accumulated to the effect point [1], which is the point that congestion affect to the CCTV's velocity obviously and this point is the main idea of the model.

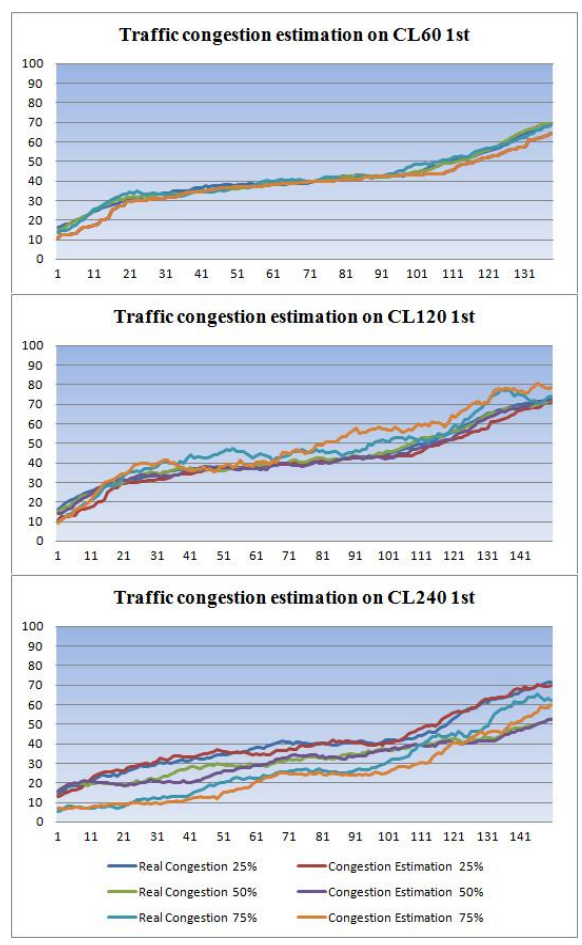

Fig. 4. Graph of traffic congestion prediction compare with real traffic congestion (All of $^{\mathrm{st}}$ experiment on $0.5 \mathrm{~km}$ road length).



Fig. 5. Graph of traffic congestion prediction compare with real traffic congestion (All of $2^{\text {nd }}$ experiment $0.5 \mathrm{~km}$ road length). 


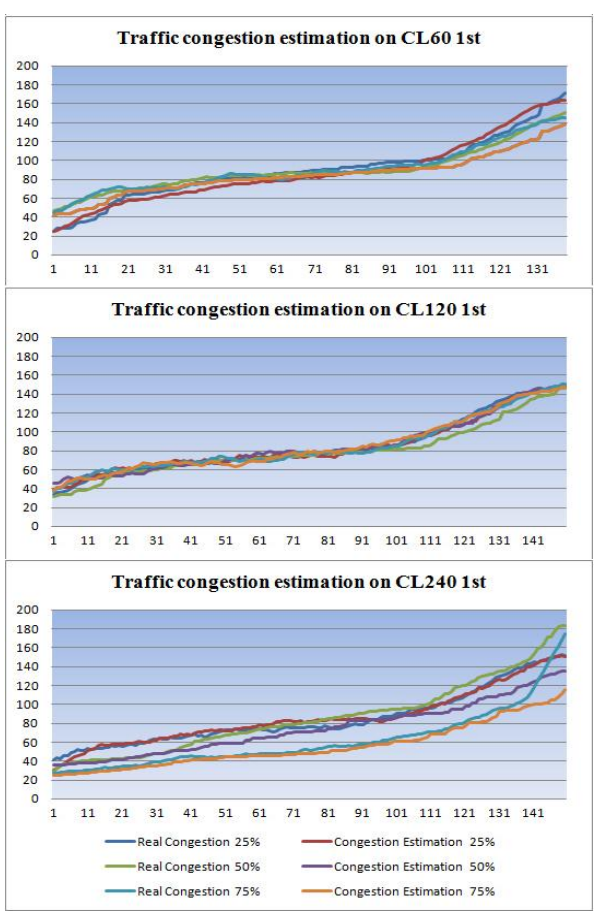

Fig. 6. Graph of traffic congestion prediction compare with real traffic congestion (All of $1^{\text {st }}$ experiment $1.0 \mathrm{~km}$ road length).

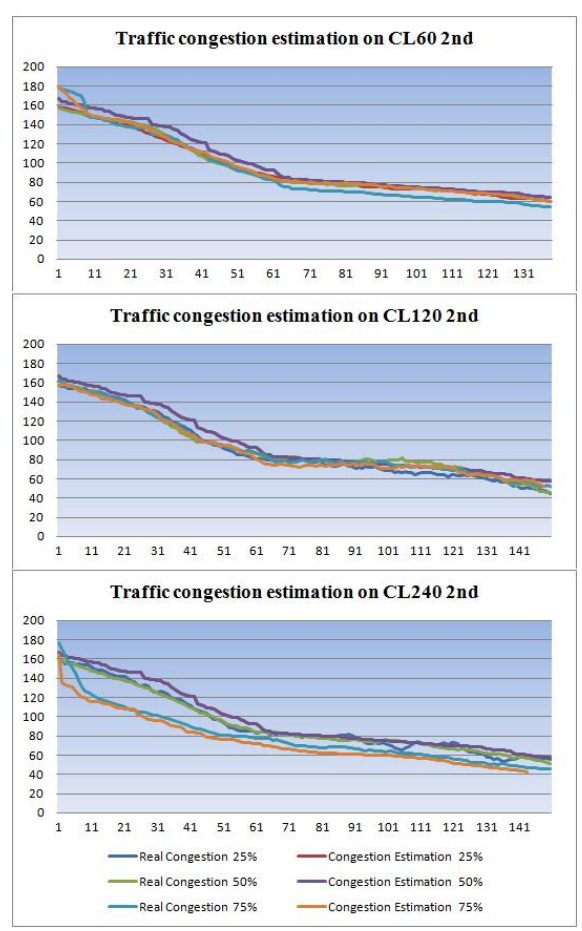

Fig. 7. Graph of traffic congestion prediction compare with real traffic congestion (All of $2^{\text {nd }}$ experiment $1.0 \mathrm{~km}$ road length).

\section{REFERENCES}

[1] A. Buranasing and A. Prayote, "Travel time estimation for highway in pre-timed systems," in Proc. The 2nd International Conference on Networking and Information Technology Hong Kong, China, 2011.

[2] B. Krause, C. V. Altrock, and M. Pozybill, "Intelligent highway by fuzzy logic: Congestion detection and traffic control on multi-lane roads with variable road signs," IEEE International Conference, 1996.

[3] Y. Ando, O. Masutani, and S. Honiden, "Performance of pheromone model for predicting traffic congestion," AAMAS'06, Hakodate, Hokkaido, Japan, 2006

[4] Q. Paramics. Traffic microscopic simulation software. (January 2, 2013). [Online]. Available: http://www.paramics-online.com.

[5] F. L. Hall, "Traffic stream characteristics," Transportation Research Board (TRB), 1992.

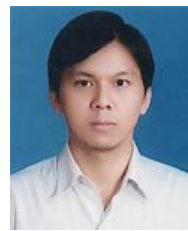

Arthit Buranasing was born in 1984 . He received B.Sc. and M.Sc. degree in Computer Science from King Mongkut's University of Technology North Bangkok, Thailand. He is a lecturer in faculty of engineering at Dhurakij Pundit University, Thailand His research interests include artificial intelligence, computer algorithms and traffic engineering.

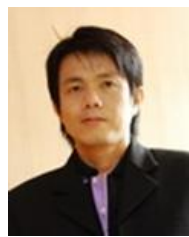

Chaiyaporn Khemapatapan was born in 1972 . He received Ph.D. degree in Electrical Engineering from Chulalongkorn University, Thailand. $\mathrm{He}$ joined a faculty of engineering as a lecturer at Dhurakij Pundit University, Thailand. His research areas are in computer networking, artificial intelligence and wireless networks. 